\title{
SONDAGGIO ACUSTICO DELLE NUBI
}

\author{
Guglielmo Zanotelli
}

Il metodo per l'esplorazione acustica delle nubi, che ci accingiamo ad esporre, si basa sullo studio del potere riflettente di queste per le onde sonore della gamma udibile, ed è diretto non alla determinazione degli elementi geometrici macroscopici, forma, dimensioni, distanza dalla superficie terrestre delle nubi stesse, ma alla conoscenza della intima costituzione, e cioè della quantità e della grandezza delle goccioline d'acqua che le compongono.

A tal fine si determina la dipendenza del coefficiente di riflessione di un banco di nubi, cioè del rapporto fra la potenza sonora riflessa e quella che vi incide normalmente, dalle suddette grandezze, e si studiano le curve rappresentative dello stesso coefficiente in funzione della frequenza sonora. In genere il coefficiente di riflessione dipende da diversi parametri legati alle condizioni dell'aria e dell'acqua comprese nella nube, ma soprattutto dalla temperatura e dalla pressione. Il calcolo teorico mostra tuttavia cle in opportune condizioni è possibile di rendersi indipendenti da molti di questi parametri, cd in ispecie da quelli più variabili, temperatura e pressione, giungendo cosi a dedurre dalla determinazione del coefficiente di riflessione, in corrispondenza a certe frequenze caratteristiche, il raggio ed il numero delle goccioline di acqua contenute nell'unità di volume della nube.

Risulta anzi che in pratica un apprezzammento sommario dell'entità di tali elementi può ottenersi dal semplice esame della forma delle curve del coefficiente di riflessione in funzione della frequenza sonora, e che inoltre, per mezzo dell'osservazione degli spostamenti che dette curve subiscono nel piano rappresentativo al trascorrere del tempo, può seguirsi la evoluzione delle goccioline costituenti la nube, sia per evaporazione che per ulteriore condensazione di vapor acqueo sulle stesse, sia per l'eventuale suddivisione di ciascuna goccia, o viceversa per la riunione di più goccioline in una sola.

Il coefficiente di riflessione $R$ entrando dall'aria secca in un banco di nubi di grande spessore, lateralmente indefinito e delimitato verso 
la libera atmosfera da una superficie piana, costituito da groccioline sferiche di acqua tutte uguali e uniformemente distribuite, e colpito dal raggio sonoro sotto incidenza normale, fu già da me determinato ( $\left.{ }^{1}\right)$ : procedetti allora dai risultati ottenuti da Oswatitsch $(-)$ ronsiderando il processo termodinamico cui è soggetta l'onda sonora in conseguenza della alterna condensazione ed evaporazione delle goccioline d'acqua; nonché da quelli da me medesimo ottenuti precedentemente $\left({ }^{3}\right)$ trattando del fenomeno meccanico dell'attrito opposto dalla viscosita dell'aria al trascinamento delle goccioline stesse nella oscillazione sonora.

Si ha approssimativamente, come mostrai a suo tempo,

$$
R \cong R_{\mathrm{c}}+R_{\mathrm{a}}
$$

essendo $R_{\mathrm{c}}$ il coefficiente di riflessione per condensazione, che ticne conto ciò̀ del fattore termodinamico, ed $R_{\mathrm{n}}$ il cocfficiente di rifles-

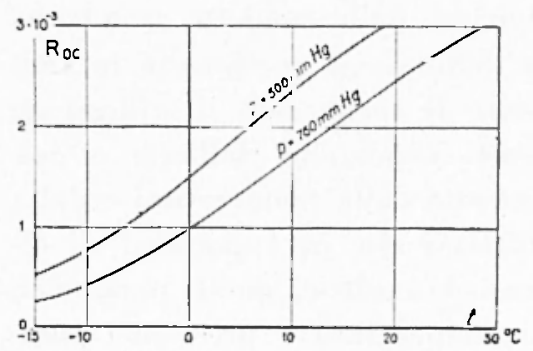

Fig. 1

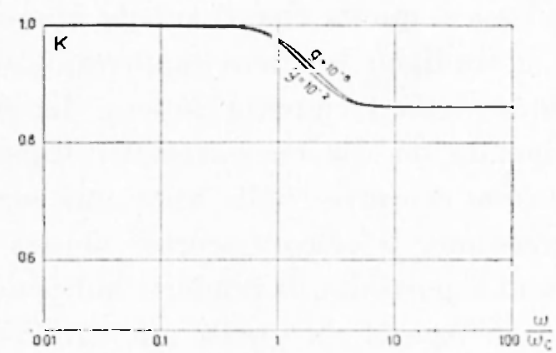

Fig. 2

sione per attrito, che tiene conto del fenomeno meccanico. Il primo dei due coefficienti è dato da

$$
R_{\mathrm{c}}=K \frac{R_{\mathrm{oc}}}{\left[1-\left(\frac{\omega}{\left(\omega_{\mathrm{c}}\right.}\right)^{2} g\right]^{2}+\left[\frac{\omega}{\left(\omega_{\mathrm{c}}\right.}\right]^{2}}
$$

ove $R_{o r}$, che è il coefficiente di riflessione per condensazione per la frequenza zero, dipende solo dalla pressione atmosferica e dalla temperatura, ed ̀̀ rappresentato nel grafico di fig. $1 . K$ è un fattore numerico dipendente invece anche lievemente dalla frequenza (1)/2 $\mathrm{dcl}$ suono, ma sempre compreso fra 1 e 0,8: il grafico di fig. 2 nt: mostra l'andamento per la temperatura $t=10{ }^{\circ} \mathrm{C}$ e la pressione $p=600$ numHg. I due parametri (1) e gr valgono poi 


$$
\begin{gathered}
\omega_{c}=\frac{q}{a^{2}} 3 D \frac{1+\gamma\left(\lambda_{\mathrm{s}}-\lambda_{\mathrm{f}}\right)}{1+\frac{a_{\mathrm{o}}}{a}+\frac{\lambda_{\mathrm{s}} c_{\mathrm{p}} \varrho D}{k}}, \\
g=q\left(1+-\frac{a_{\mathrm{o}}}{a}\right) \frac{c_{1} \varrho_{1} D}{k} \frac{1+\gamma\left(\lambda_{\mathrm{s}}-\lambda_{\mathrm{f}}\right)}{\left(1+\frac{a_{\mathrm{o}}}{a}+\frac{\lambda_{\mathrm{s}} c_{\mathrm{p}} \varrho}{k}\right)^{2}} .
\end{gathered}
$$

In queste formule $q \dot{e}$ il rapporto fra il volume occupato nella nuhe dall'acqua contenutavi sotto forma di gocce e rispettivamente dall'aria; $\varrho, k, c_{\mathrm{p}}, \gamma$ rappresentano rispettivamente la massa specifica, il coefficiente di conducihilità termica, il calore specifico a pressione costante ed il rapporto di quest'ultimo al calor specifico a volume costante per l'aria; $\varrho_{1}, c_{1}$ la massa specifica ed il calor specifico del-

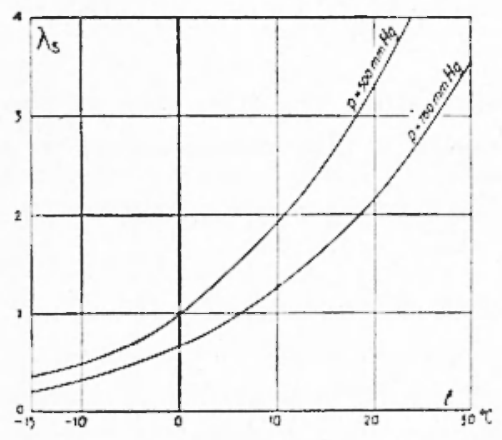

Fig. 3

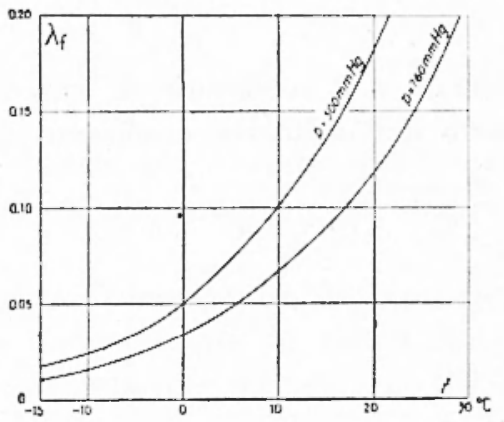

Fig. 4

l'acqua; $D$ il coefficiente di diffusione del vapor acqueo nell'aria, $a$ e $a_{0}$ il raggio delle goccioline ed un raggio caratteristico nel processo di accrescimento delle stesse per condensazione, che può ritenersi, per le normali circostanze metereologiche alle nostre latitudini, essere $a_{0}=5.10^{-4} \mathrm{~cm}$; infine $\lambda_{,} \mathrm{e}$ i. sono due funzioni della temperatura e della pressione rappresentate dai grafici delle figure 3 e 4 riprodotti dal lavoro di Oswatitsch.

11 grafico di fig. 5 descrive l'andamento del rapporto $R_{\mathrm{c}} / R_{\mathrm{oc}}$ in funzione di $\omega / \omega_{c}$ per la pressione di $600 \mathrm{mmHg}_{\mathrm{g}}$ e la temperatura di $10{ }^{\circ} \mathrm{C}$. Come si vede, il coefficiente di riflessione per condensazione si mantiene praticamente uguale a $R_{o c}$ dalla frequenza zero sino in prossimità di $\omega_{\mathrm{o}} / 2 \pi$ : è questa una frequenza di taglio, oltre la quale il potere riflettente decade rapidamente; per $n=\omega_{c}$ si ha all'incirca $R_{\mathrm{c}} \simeq 0,5 R_{\mathrm{oc}}$. II valore di $g$, che in realtà $\grave{e}$ sempre piccolo, al massimo qualche centesimo, se si tiene presente che in pratica si ha al 
più $q=10^{-5}$ in corrispondenza al contenuto assai rilevante di $10 \mathrm{~g}$ di acqua per $\mathrm{m}^{3}$ di nube, influenza solo in misura ridotta l'ultimo tratto della curva del coefficente di riflessione, ove questo è già molto piccolo.

Il coefficiente di riflessione per attrito è espresso con grande approssimazione da

$$
R_{\mathrm{a}}=\left(\frac{q(\varepsilon-1)}{4}\right)^{2} \frac{\left(\frac{2(\varepsilon-3)}{9(\varepsilon-1)} z+1\right)^{2}+\left(1+\frac{1}{z}\right)^{2}}{\left(\frac{2}{9}(2 \varepsilon+1) z+1\right)^{2}+\left(1+\frac{1}{z}\right)^{2}}
$$

ove i parametri $\varepsilon, z$ hanno i valori

$$
\varepsilon=\frac{\varrho_{i}}{\varrho}-1 \quad, \quad z=a \sqrt{\frac{\omega}{2 y}},
$$

essendo $v$ il coefficente di viscosita cinematico dell'aria, cioè il rapporto dell'ordinario coefficente di viscosità $u$ alla massa specifica $\varrho$.

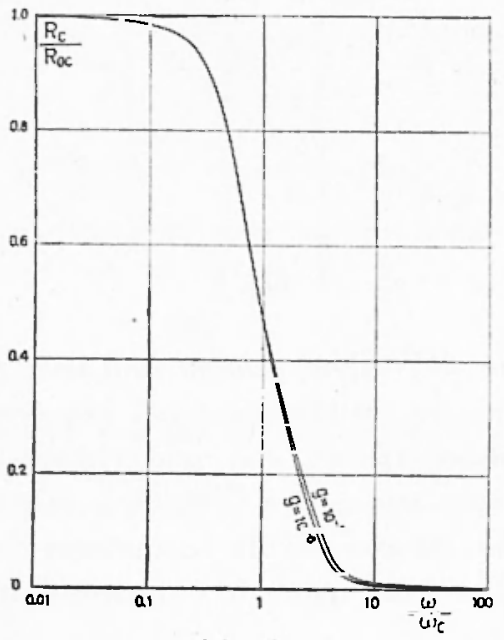

Fig. 5

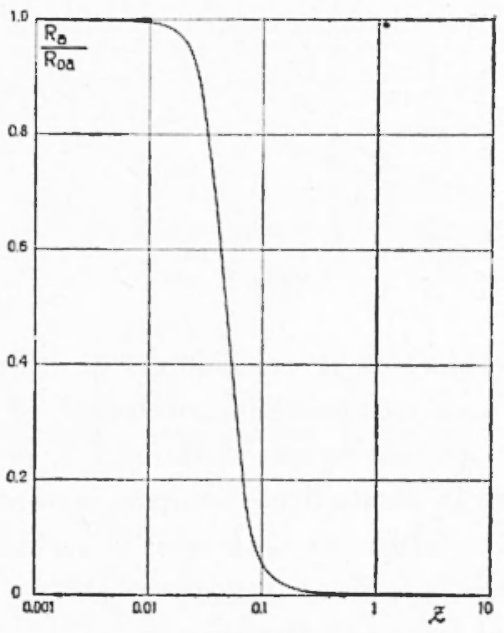

Fig. 6

Trascurando numeri dell'ordine dell'unità difronte ad $\varepsilon$ che è circa $10^{3}$, si ha più semplicemente

$$
R_{\mathrm{a}} \cong\left(\frac{q \varepsilon}{4}\right)^{2} \frac{\left(\frac{2}{9} z+1\right)^{2}+\left(1+\frac{1}{z}\right)^{2}}{\left(\frac{4 \varepsilon}{9} z+1\right)^{2}+\left(1+\frac{1}{z}\right)^{2}} .
$$


II fattore

$$
R_{\mathrm{oa}}=\left(\frac{q(\varepsilon-1)}{4}\right)^{2} \cong\left(\frac{q \varepsilon}{4}\right)^{2}
$$

è il coefliciente di riflessione corrispondente alla frequenza zero; pur assumendo $q=10^{-5}$, si ha per $R_{0 a}$ il valore assai modesto $6,25,10^{-6}$.

L'andamento del rapporto $R_{\mathrm{a} 1} / R_{\text {ol }}$ è espresso in funzione di $\tilde{z}$ del grafico di fig. 6 e rassomiglia nel primo tratto a quello dell'analogo rapporto per condensazione. Come frequenza di taglio può essere assunta ora quella per cui

$$
z=\frac{3}{2 \sqrt{\varepsilon}}
$$

che corrisponde alla pulsazione

$$
\omega_{\mathrm{a}}=\frac{1}{a^{a}} \frac{9 v}{2 \varepsilon} \approx \frac{1}{a^{n}} \frac{9 u}{2 \varrho_{1}} ;
$$

a tale frequenza ̀̀ circa $R_{\text {، }} \cong 1,5 R_{0 \%}$. Invece per z molto grande questo coefliciente di riflesione tende al valore costante e piccolissimo $R_{\mathrm{n}} \sim(q / 8)^{2}$.

Le due frequenze di taglio per condensazione e rispettivamente per attrito rivestono importanza fondamentale ai nostri fini e quindi ci interessa di studiarle più attentamente. Secondo la [3] la $\omega_{c}$ è proporzionale al rapporto $q$ fra i volumi dell'acqua e dell'aria nella nuhi, e diminuisce al crescere del ragrio delle roccie; la ${ }^{\prime \prime}{ }_{a}$ invece secondo la [10] $\dot{e}$ indipendente da $q$ ed inversamente proporzionale al quadrato del raggio delle goccie. Riferiamoci ai già indicati valori di una pressione atmosferica di $600 \mathrm{mmHg}$, ed una temperatura di $10^{\circ} \mathrm{C}$ : in queste condizioni l'andamento delle due frequenze di tagrlio in funzione del ragrgio delle gocee risulta dalla fig. ?. Le stesse risultano sempre assai diverse fra loro: per il valore massimo $q=10^{-5}$ la $\omega_{a l}$ è poco'meno che un centinaio di volte magriore della (1): tale intervallo cresce ancora per valori minori del contenuto di acqua.

Passiamo ora ad esaminare quali possono essere gli eflettivi elementi su cui hasare il sondagrgio acustico delle nuhi:

I) Il rilevamento dei due coefficienti di riflessione temodiuamico e rispettivamente meccanico, per una o più frequenze dei relativi campi di azione, e la deduzione delle caratteristiche delle gocce attraverso le formule [2] e [5]. 
2) La determinazione delle frequenze di taglio $(t) / \bar{\alpha} \pi$ e $\omega_{\mathrm{a}} / 2 \pi$ per mezzo del rilevamento del coefficiente di riflessione in due bande di frequenza circoscritte, e la loro interpretazione con le [3] e [10].

3) Il rilevamento dell'andamento del coefficiente di riflessione in funzione della frequenza in una estesa banda di frequenze e la deduzione delle caratteristiche degli elementi costitutivi della nube dalla forma delle curve relative.

Discutiamo separatamente queste tre possibilità che si presentano

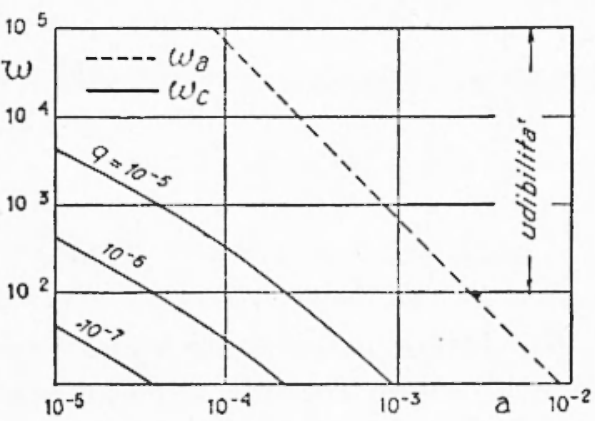

Fig. 7 per il nostro fine. 11 rilevamento del coefficiente di riflessione consiste nel'appre\%zare l'entiti della energia riflessa in rapporto a quella incidente alla superficic del. lo strato nuvoloso: il valore di questo rapporto non coincide certo con quello, rilevabile alla superficie terrestre, fra energia emessa dal trasmettitore delle onde sonore e ricevuta dal ricevitore. La determinazione quantitativa richiede la esatta conoscenza dei rendimenti di questi organi, questione che rientra sostanzialmente fra quelle di natura sperimentale, nonché degli angoli solidi da considerare alla trasmissione ed alla ricezione, cioc delle caratteristiche di direttività del trasmettitore e del ricevitore; c’è anche da considerare l'assorbimento atmosferico nel percorso di andata e ritorno del raggio sonoro. Evidentemente la misura, diciamo cosi assoluta, del coefficiente di riflessione per una data frequenza i legata alla incertezza di tutti questi elementi, e presenta perciò difficoltà di gran lunga superiori a quelle del rilevamento delle frequenze di laglio, o dello studio qualitativo dell'andamento del coefficiente di riflessione al variare della frequenza. È da ritenere percio che, salvo circostanze particolari, questa prima via non si presti ad una agevole attuazione, e pertanto su questo punto non ci soffermiamo ulteriormente.

Il rilevamento delle frequenze di taglio richiedle l'esplorazione di una gamma più o meno larga di frequenze, ed in sostanza non va disgiunto dalla terza possibilità, quella del rilevamento della curva 
del coefficiente di riflessione in funzione della frequenza. Si tratta in pratica di predisporre un trasmettitore a frequenza sonora variabile, ed un ricevitore che possa misurare il rapporto fra i segnali ricevuti, dirctamcnte e rispettivamente per riflessione. La realizzazione sperimentale con apparecchiature elettroacustiche può prevedere una trasmissione ad impulsi, con treni di onde sonore sinusoidali, dato il tempo notevole intercorrente fra la ricezione dell'impulso diretto e di quello riflesso a causa della hassa velocita del suono nell'atmosfera. Oppure potrebbe pensarsi all'emissione di un impulso aperiodico, ed all'esame della forma del segnale ricevuto dopo la riflessione, per ricavarne i rapporti di attenuazione delle varie componenti armoniche; ma questa seconda via si presenta più ardua sia dal punto di vista teorico che da quello sperimentale.

Conosciuta la frequenza di taglio per attrito $\omega_{\mathrm{a}} / 2 . \tau$ è immediatamente determinabile, per mezzo della [10], il raggio delle goccioline riflettenti. Poiché il coefficiente di viscosità cinematico è inversamente proporzionale alla massa specifica dell'aria, $v=\mu / \rho$ ove $\mu$ e lordinario coefficiente di viscosità, e poiché è molto prossimamente $\varepsilon=\rho_{1} / \rho_{\text {, }}$ la [10] risulta praticamente indipendente dalla pressione atmosferica, e solo dipendente dalla temperatura in quanto ne dipendono il coeffciente di viscosità e la massa specifica dell'acqua; peraltro l'effecto della temperatura, il cui intervallo di variazione è nella realtà limitato a poche diecine di gradi, è relativamente tenue per ambedue queste grandezze, sì da influenzare ben poco la determinazione del raggio delle goccioline con la relazione

$$
a \subseteq \sqrt{\frac{1}{\omega_{\mathrm{a}}} \frac{9 \mu}{2 \varrho_{1}}}
$$

direttamente ricavabile dalla [10]. Conosciuto il raggio a si passa con la [3] alla determinazione del volume di acqua contenuto per unità di volume di nube per mezzo della conoscenza della frequenza di taglio per condensazione, o meglio servendoci della espressione del rapporto fra le due frequenze di taglio

$$
\frac{\omega_{\mathrm{c}}}{\omega_{\mathrm{a}}} \cong q \frac{2 \varrho_{\mathrm{L}} D}{3 u}-\frac{1+\gamma\left(\lambda_{\mathrm{s}}-\lambda_{\mathrm{f}}\right)}{1+\frac{a_{\mathrm{o}}}{a}+\frac{\lambda_{\mathrm{u}} c_{\mathrm{p}} \mathrm{g} D}{k}}
$$

che dipende in genere solo lievemente dal raggio delle goccie, se queste non sono piccolissime. In questa formula incide più che altro la variazione della pressione sul valore della $D$, che le è all'incirca inver- 
samente proporzionale, mentre l'effetto è minore per la $\lambda_{\mathrm{s}}$, la cui variazione ayisce nello stesso senso al denominatore ed al numeratore. Comunque per tener conto della influenza esatta della pressione e della temperatura è necessaria la loro preventiva conoscenza, da desumersi per altra via.

Una volta note le grandezze a e $q$ risulta determinato anche il numero $N$ delle goccioline contenute per unità di volume di nulbe, per mezzo della ovvia relazione intercorrente tra queste tre grandezze

$$
a=\frac{4}{3} \pi a^{3} N
$$

Risulta sin da ora evidente come la determinazione del raggrio delle gocee sia più agevole di quella del loro numero, ciò̀ della quantità di acqua contenuta nell'unità di volume della nube. In realtà la determinazione di queste ultime grandezze, collegata alla determinazione di (1)r, è più difficile anche perché spesso la $11 . / 2 \pi$ cade al disotto della frequenza limite inferiore di udibilità per l'orecchio umano, come può vedersi dalla fig. 7. Viceversa per goccic assai piccole può cadere al disopra della frequenza limite superiore di udibilita la $(1)_{a} / 2 \pi$. ed allora il sondagrio diventa problematico. Nel primo dei due casi, ove la $\omega_{c}$ risulti eccessivamente hassa, un criterio di giudizio per stahilire la $q$ potrebhe essere dato dall'apprezzamento del coefficiente di riflessione $R_{0,1}=(q \varepsilon / 4)^{2}$; ciò una riflessione intensa sareble indizio di forte contenuto di acqua. A questo criterio vanno però applicate le stesse riserve, circa l'influenza dei fattori estranei sulla determinazions quantitativa del coefficiente di riflessione, cui abhiamo già accennato.

II rilevamento dell'andamento del coefficiente di riflessione in funzione della frequenza richiede una disposizione sperimentale dello stesso tipo di quella prevista per il rilevamento delle frequenze di taglio, e ciò trasmettitore a frequenza variabile nel campo di udibilità e ricevitore con dispositivo di misura del rapporto fra segnale diretto e segnale riflesso. Si tratta poi, data la curva del coefficiente globale di riflessione ottenuta sperimentalmente, di risalire alle caratteristiche degli elementi costitutivi della nube. $\mathrm{Ci}$ occorre perviò innanzitutto di stahilire l'andamento teorico del coefficiente di riflessione complessivo in funzione della frequenza a partire dai due coeffi-

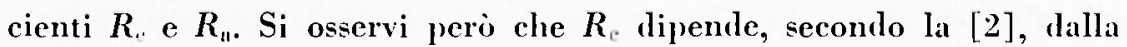
$q$ e dalla $a$ in modo complicato attraverso alle a. e g, parametri che a lor volta dipendono dalla pressione e dalla temperatura, mentre $R_{a}$ 


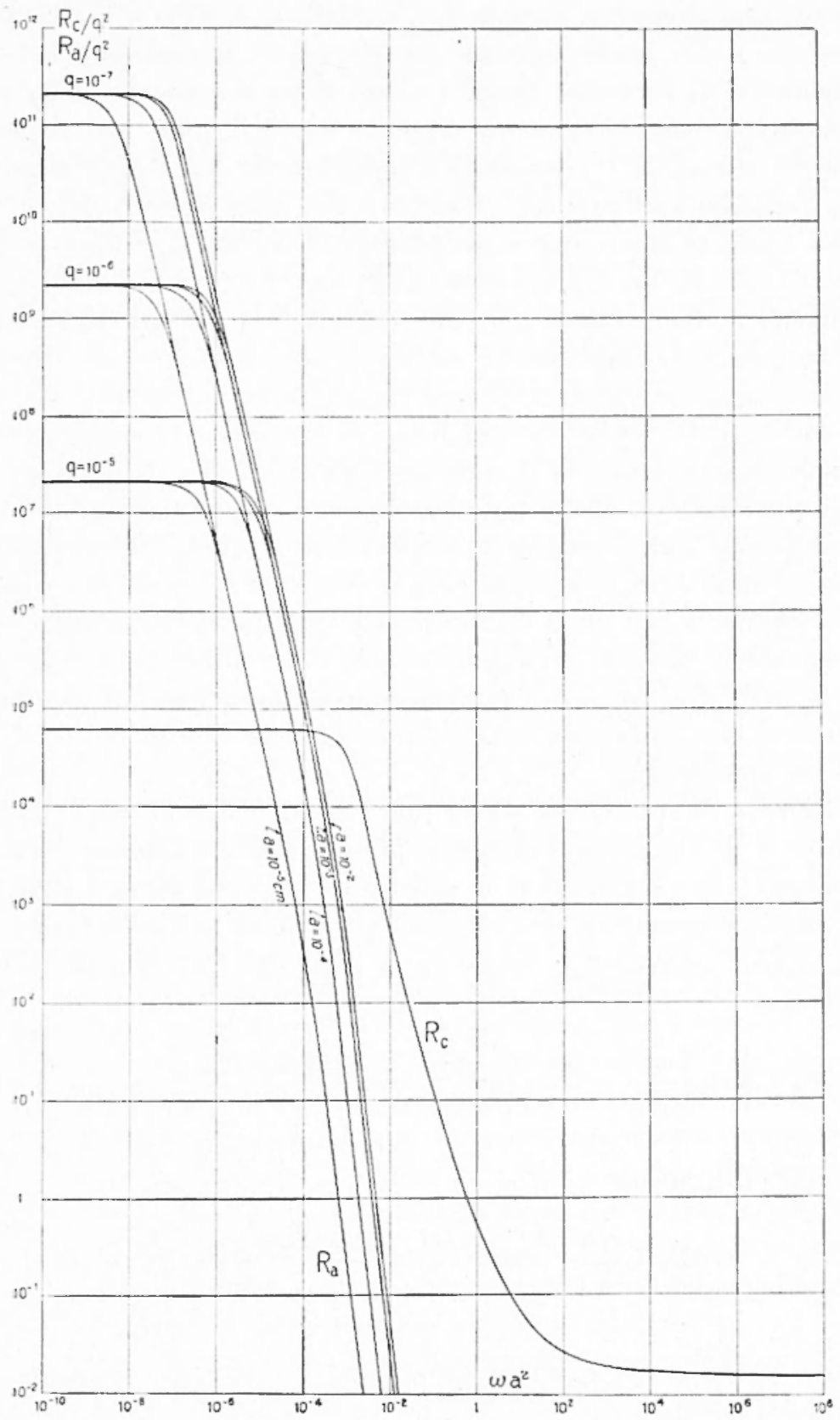

Fig. 8 
dipende esplicitamente, secondo la [5], dalla $q$, c dalla a invece attraverso alla $z$. Un modo semplice per procedere al conlronto dei due coefficienti $\hat{i}$ il seguente: fissati i valori della pressione e della tenperatura tracciamo le curve del rapporto $R_{c} / q^{2}$, il quale per frequenze superiori alla $\omega_{\mathrm{c}} / 2 \pi$ i pratieamente indipendente dalla $q$, in funzione della varialide ausiliaria $a^{*}$ : traceiano poi sullo stesso foglio la curva del rapporto $R_{a} q^{2}$, che $\dot{e}$ pure indipendente da $q$, sempre in funzione di are. In fig. 8 è riportato il grafico ottenuto cosi nelle solite condizioni di temperatura e di pressione 10 " $\mathrm{C}$ e 600 mmLtr, avende usufruito di scale logaritmiche sia per le ascisse che per le ordinate.

Dall'esame di questo diagramma appare che per un primo tratto, nell'ambito delle frequenze più hasse, il fenomeno termodinamico i nettamente prevalente, ed il coeffieiente di riflessione complessivo vitne a coincidere con quello per condensazione. Segue poi un intervallo di transizione dal fenomeno termodinamico a quello meccanico, at-

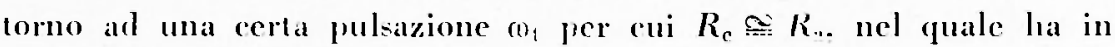
realta effetto la [1] ed il coefüiciente di riflessione risulta dalla somma dei due coefficienti per condensazione e per attrito. Infine per frequenze più alte prende definitivamente il sopravento il fenomeno di attrito, che determina praticamente da solo il coeficiente di riflessione complessivo.

La zona di transizione separa due regrioni in cui le eurve dei due coefficienti di riflessione procedono pressappoco rettilineamente nella rappresentazione logaritmica, in quanto grli stessi coefficienti risultano all'incirea inversamente proporzionali al quadrato della frequenza sonora. Infatti allorché è $\omega_{c}<<\omega<<\omega_{c} / g$ dalle [2]. [3] -i ottiene semplicennente

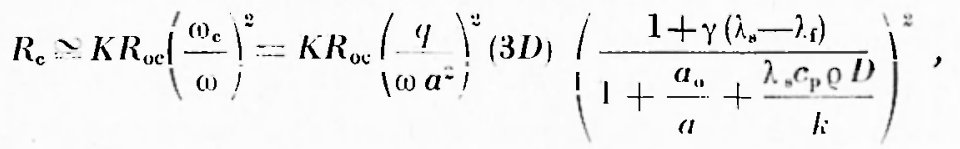

o. per la [13], anclie

$$
R_{c} \cong K R_{0 e}\left(\frac{a N}{\omega}\right)^{2}(4 \pi l)^{2}\left(\frac{1+\gamma\left(\lambda_{\mathrm{H}}-i_{\mathrm{f}}\right)}{1+\frac{a_{o}}{a}+\frac{\lambda_{\mathrm{s}} c_{\mathrm{p}} \rho}{l}}\right)^{2}
$$

Quando invece è $\omega_{\mathrm{a}}<<\omega<<4 E(1) \mathrm{a} / 9$ dalle $[6],[7], 110 \mid$ si ricava approssimativamente

$$
R_{\mathrm{a}} \simeq R_{\mathrm{oa}}\left(\frac{\omega_{\mathrm{a}}}{\omega}\right)^{2}=\left(\frac{9 v}{8}\right)^{2}\left(\frac{q}{\omega a^{2}}\right)^{2},
$$


o, per la [13], anche

$$
R_{\mathrm{a}} \cong\left(\frac{3 \pi v}{2}\right)^{2}\left(\frac{\mathrm{a} N}{\omega}\right)^{2} .
$$

Si rilevi la scarsa influenza esercitata nel valore [1t] del coefficiente di riflessione per condensazione dalla presenza, nel denominatore dell'ultimo fattore, del ragrio delle goccioline, almeno sinché quest'ultimo non è molto piccolo. Se ne deduce che il coefficiente di riflessione complessivo viene ad cssere, nelle due regrioni contique alla transizione, all'incirca proporzionale allat frandezza $1 q / \omega) a^{-}{ }^{-}$od anche alla grandezza $(n N / \omega)$. I aver messo in luce questa circostanza ci tornera utile in seguito per la nostra discussione.

Sommando le ordinate nel grafico di fig. 8 si ottiene, secondo la [1], la $R / q^{2}$, ma praticamente questa curva viene a coincidere, nella rappresentazione logaritmica, con le curve di $R_{r} q^{-}$nel primo tratto e di $R_{a} q^{-}$nell'ultimo, distaceandosene sensibilmente solo nella regione di transizione. Cambiando poi le unita di misura sugrli assi coordinati possono ricavarsi le curve $R(\omega)$. Possiamo quindi sin da ora prevedere che in queste nuove curve si distingueranno $i$ seguenti tratti caratteristici : un primo tratio orizzontale, per' $\omega<<\omega_{c}$, in cui $R \simeq R_{w}$, indipendente da $q$ e da $a$, dipende solo dalle condizioni ambienti di temperatura e di pressione; la regione del womito della curva di condensazione attorno alla pulsazione di taglio $\omega_{\mathbf{c}}$; il tratto quasi rettilinea per condensazione, allorehe $\left(\omega_{c}<<\omega<<\left(0_{t}\right.\right.$, in cui valgono le [14]. [15]; la regrone di transizione dal regime termodinamico a quello meccanico, sino al gomito della curva per attrito, attorno alle pulsalzioni $\omega_{1}$ ed $\omega_{a}$; il tratto quasi retilineo per attrito, per cui $\omega_{a}<<\omega<$ l $\varepsilon \omega_{4}, 9$, in cui valgono le $[16],[17]$; infine, per frequenze maggiori. il tratto in cui il coefficiente di riflessione tende al valore costante $14 / 8 \%^{\circ}$. Se si fa astrazione dal primo tratto orizzontale, il coefficiente di rifessione risulta sempre più o meno esattamente proporzionale a $q$. il che significa che al variare di $q$, per a costante, la curva rappresentativa subisce una traslazione in direzione verticale, alterandosi solo lievemente nella forma. Invece la variazione di $a$, per $q$ costante, determina uno spostamento in senso orizzontale e contemporaneamente varia l'ampiezza della regione compresa tra $\omega_{1}$ ed $\omega_{a}$.

Nella realta solo una parte relativamente esigua della curva $R(\omega)$ vicne a cadere nell'ambito delle frequenze udibili, ciós solo un particolare tratto di tale curva, differente a seconda del valore di $a$. potrà venire osservato sperimentalmente, e sarà utilizzabile ai fini 
del sondaggio. Nella fig. 9 sono riportate appunto le varie curve che si desumono dal wrafico di figr. 8 per $R$, esprimendo in ascisse le $\omega$ invece delle wa ${ }^{2}$, e prendendo in considerazione le sole frequenze comprese entro la gramma di udibilita. Come si vede, dall'aspetto delle

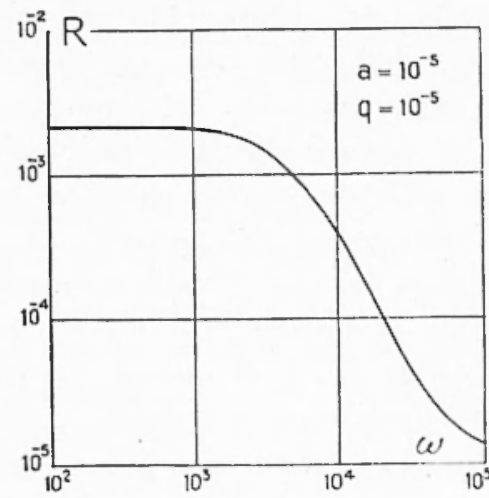

Fig. 9a

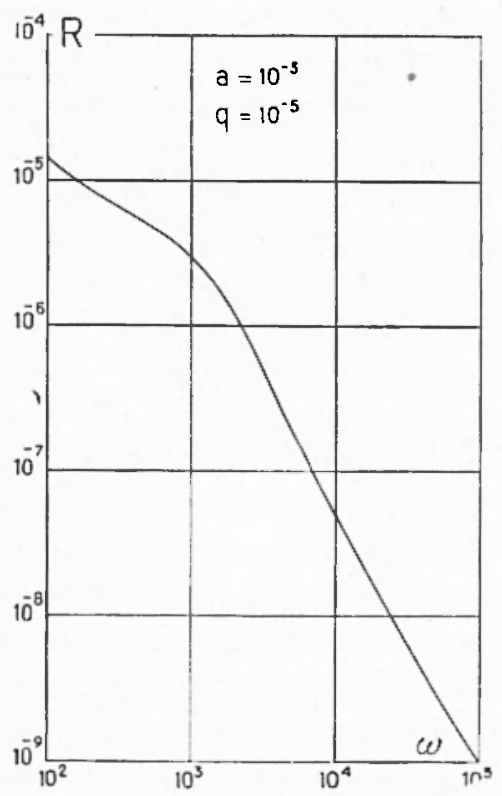

Fig. 9c
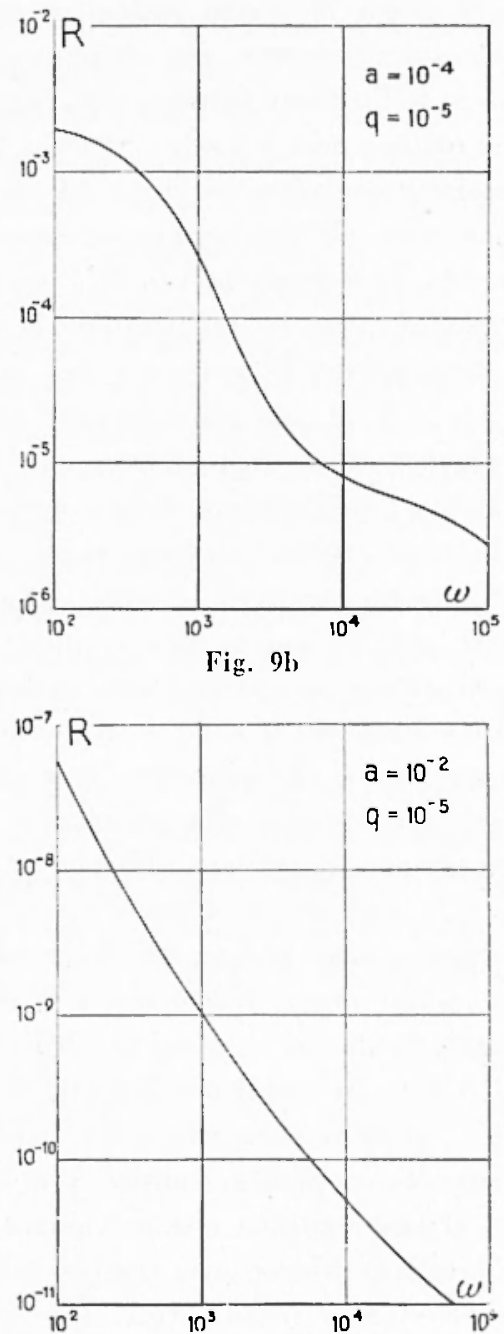

Fig. 9d

curve $R(\omega)$ è possibile dedurre il raggio delle gocce: per $a=10^{-5}$ cm (figr. 9a) si ha la semplice caratteristica di un filtro passa-basso; per $a=10^{-4} \mathrm{~cm}$ si palesano simultaneamente (fig. 9l,) ambedue le frontiere di taglio in $\omega_{c}$ ed $\omega_{\mathrm{n}}$; per $a=10^{3} \mathrm{~cm}$ cade ormai (fig. 9c) 
nel campo di udibilita la sola $\omega_{\mathrm{n}}$; infme per $a=10^{-2} \mathrm{~cm}$ ambedue le frequenze di tagrlio sono (fig. 9d) al disotto dell'udibilità ed il fattore di riflessione tende. al crescere della frequenza, al valorc costante gia detto. Le curve ora mostrate sono quelle relative a $q=10^{-5}$, ma diminuendo od aumentando quest'ultimo valore non si viene ad alterame sostanzialmente l'aspetto, ma solo a distanzare più o meno la posizione delle frequenze di taglio. E questa una caratteristica rilevante, che l'aspetto del tratto della curva $R(\omega)$ che cade nell'udibilita dipende principalmente dal raggio delle goceie e non dalla quantita di acqua contcnuta nell'unita di volume di nube: quest'ultimo elemento si potreble arguire dalla maggiore o minore distanza in ordinate delle due frontiere di taglio ove simultaneamente rilevaljili: in pratica una rolta interpretato qualitativamente il grafico con un esame sommario, la determinazione di a e di $q$ può farsi dai valori di $\omega_{a}$ e del rapporto $\omega_{c} / \omega_{a}$ per mezzo delle $[11],[12]$, od anche, salvo le riserve fatte precedentemente, con la $[8]$ dal valore di $R_{\text {on. }}$.

Per la costruzione del grafico ora descritto e per la relativa discussione, ci siamo riferiti per semplicita ad una particolare condizione di temperatura e di pressione. E logrico chichersi ora se le conclusioni raccolte possano estendersi a condizioni diverse, cioè particolarmente se in tal caso vengrano conservate le caratteristiche delle curve del coefficiente di riflessione, specie nel tratto relativo al fenomeno di attrito che è il più interessante ai fini del sondagrio acustico. Osserviamo a questo proposito che il coefficiente per attrito potrel,he renir mascherato da quello per condensazione anche oltre la pulsazione di tagrlio $\omega_{n}$, se nel grafico di fig̣. 8 la curva per condensazione, pur conservando approssimativamente la sua propria forma suluisse

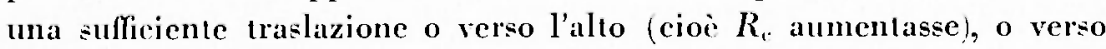
destra (ciò̀ aumentasse $\omega_{c}$, e ciò tanto più facilmente quanto mang. giore fosse il raggio $a$.

Consideriamo allora nelle espressioni [3] della pulsazione di tagrlio per condensazione e [1+] del relativo coefficiente di riflessione il ruolo del fattore $\left[1+\gamma\left(\lambda_{\mathrm{s}}-\lambda_{\mathrm{f}}\right)\right]\left[1+\left(a_{\mathrm{o}} / a\right)+\left(\lambda_{\mathrm{g}} c_{\mathrm{p}} \mathrm{Q} D / k\right)\right]$. Per l'aria il coefficiente di diffusione è dato con huona approssimazione, in unita C.G.S., da $D=0,22[1+(t / 273)]^{1, .5} .760 / p>0,22(1+0,0061 t) .760 / p$; quindi il prodotto ${ } D$ risulta indipendente dalla pressione, e solo lievemente dipendente dalla temperatura. Cosi è anche allincirca per il rapporto $c_{1} /: \sim 4.10^{*}$, e quindi la grandezza $c_{\mathrm{p}} D / h$ si mantiene 
pressappoco costante e di valore non molto diverso da $\gamma=1,49$. Ne deriva, tenuto presente il fatto che $\dot{c}$ sempre $\lambda_{n}>>\lambda_{f}$, che nella condizione piu sfavorevole, quando $a_{0} / a<<$, il fattore che a noi interessa si mantiene prossimo all'unità, limitandosi le variazioni apportate dalle ammissiluli variazioni di temperatura e di pressione atmosferiche ad alcuni centesimi del suo valore. Secondo la [14] il coefficiente di riflessione per condensazione risulta allora all'incirca proporzionale al quadrato del coefficiente di diffusione, e cioe inversamente proporzionale al quadrato della pressione, e pochissimo dipendente dalla temperatura.

Il coeffeiente di viscosità per l'aria puo essere d'altra parte espresso con la formula empirica di Grindley e Gilson $u=0,00017(1+t$ $+0,(00329 t)$ ed $\dot{e}$ praticamente indipendente dalla pressione, cosi che $v=\mu / Q$ risulta inversamente proporzionale a questultima: secondo la [16] anche il coefficiente di riftessione per attrito i cosi inversamente proporzionale al quadrato della pressione e solo lievemente dipendente dalla temperatura.

Da tutto ciò deriva che mentre le variazioni di temperatura, limitate in pratica a poche decine di gradi, possono produrre qualche lequero spostamento della posizione reciproca dei tratti retlilinei e paralleli dei due coeffieienti di riflessione in rappresentazione logaritmica, anche notevoli variazioni di pressione non hamno, a parita di valore della grendezza $R_{w}$, altro effetto che di spostare insieme i suddetti tratti mantenendone costante la distanza. Tale distanza viene in effetti a dipendere dalla temperatura e dalla pressione solo per il tramite di $R_{\text {un', }}$ in quanto e per l'unico fatto che questa prandezza ne dipende. Possiamo domandarei quale sarehbe la condizione limite per cui il tratto rettilineo del coefficiente di riftessione per condensazione venisse ad allinearsi ed a costituire la prosecuzione del tratto rettilineo del coefficiente per attrito, dato che, come $s$ ovvio, i due tratti hamno già la stessa pendenza nel diagramma logarimico $R\left(r_{0}\right)$, essendo ambedue i coeffreienti inversamente proporzionali al quadrato della frequenza sonora. Tale condizione si stabilisce immediatamente imponendo l'uguaglianza dei due coefficienti per una stessa generiea pul. sazione, ció̀ uguagliando la [16] alla [14], nella quale l'ultimo fattore può essere preso semplicemente uguale ad uno. Così facendo risulta per $R_{w}$ un valore dell'ordine di $10^{1}$, mentre nella realta e secondo quanto appare dal wrafico di fig. 1 , anche nelle pegriori condizioni, temperatura alta e pressione hassa, non si arriva neppure al valore $10^{\circ}$. Ciò significa che, pur con i magrogiori valori di $R_{0 \times}$ ammis- 
sibili in pratica il tratto rettilineo per condensazione si mantiene sempre a sinistra di quello per attrito, cioe che il cocfficiente di riflessione per condensazione non arriva mai a mascherare, per $(0)>\omega_{a}$ il cocfliciente per attrito, e che quindi l'aspetto delle curve $R(1$,$) non$ viene sostanzialmente modificato nemmeno nelle più sfavorevoli condizioni di temperatura e di pressione.

Alla stessa conclusione si arriva considerando la [12], ed imponendo la condizione $\omega_{\mathrm{e}} / \omega_{\mathrm{a}}=7$, cioi che la frequenza di taglio per condensazione raggiunga quella per attrito: pur prendendo il valore massimo ammissibile $q=10^{-\overline{-}}$, necessitereblye che il coefliciente di diffusione $D$ risultasse un centinaio di volte maggiore del normale, cioè che la pressione si riducesse a valori corrispondentemente hassi ed inammissibili in pratica.

All'inizio di questo nostro lavoro abbiamo posto la esplicita premessa di considerare la riflessione su un hanco di nubi di grande spessore: ció era determinato dalla necessita che si potessero trascurare le riflessioni all'uscita del hanco, o su eventuali strati successivi. Nella realta l'assorlimento dell'onda sonora nell'interno delle nulbi è abloastanza forte, specie per il fenomeno di attrito ed alle frequenze elevate, da assicurarci che questa esigenza possa essere soddisfatta anche con spessori morlerati. Ma se per l'osservazione sperimentale è effottivamente adotata la trasmissione ad impulsi da noi avanti prevista, pli stessi intervalli di tempo, intercorrenti fra i segnali originati dalle riflessioni su eventuali superfici di discontinuita sucessive a ¡uella di ingresso, appaiono sufficienti a riconoscerle e ad individuarle.

Prima di procedere $\dot{e}$ anche opportuno considerare due circostanze che potrebbero sugrgerire qualche dubbio sulla piena applicalibitit dei risultati desunti dalla teoria termodinamica di Oswatitsch. La prima di queste discende dalla osservazione che per il calcolo del coefficiente di riflessione per condensazione si parte dall'indice di ritrazione che presenta un banco di nulbi, entrandovi dall'aria secca: ora non è detto che in prossimitì della superficie d'ingresso la condizione dellassenza di umidita dall'aria sia soddisfatta: se cosi non fosse si avrebbe un riavicinamento dell'indice di rifrazione all'unita, ed una conseguente diminuzione del coeffieiente di riflessione. Tuttavia non verreblye a spostarsi la frequenza di taglio $\left(n_{c} / 2 \pi\right.$ che dipende solo dalle caratteristiche del mezzo riflettente e non da quelle del mezzo d'ingresso, e quindi resterehbero in vigore le determinazioni hasate sul rilevamento di tale frequenza. 
Il secondo punto riguarda una oliezione sollevata dallo stesso Oswatitsch in appendice al suo lavoro, circa la validità quantitativa dei risultati da lui ottenuti per goccie piccole, di raggio inferiore a $10^{-4} \mathrm{cu}$, in conseguenza della mutata forma della legre di accrescimento delle goccie nel processo di condensazione. Tale riserva deve venir conservata anche nei riguardi del presente metodo, per la parte direttamente derivante dalla teoria termodinamica, e per goccie di raggio molto ridotto.

Soffermiamoci anche brevemente a considerare l'effetto, ai fini del sondaggio acustico, della eventualità che le goccioline costituenti ciascun elemento di volume del hanco di nulji non siano tutte dello stesso raggio. Rinunciando ad ogni pretesa di apprezzamento rigoroso, che implicherelibero naturalmente una nuova impostazione teorica, possiamo procedere ad una valutazione sommaria ammettendo la semplice sovrapponiluilità degli effetti prodotti dalle diverse aliquote $q$ dell'acqua costituente le goccie dei vari raggi $a$, contenuta nell'unità di volume di nulse.

Riferiamoci al caso tipico che dette aliquote siano tutte uguali per i vari raggi delle goccie, condizione questa che non dovrebbe essere troppo discosta dalla realtà nell’ipotesi che le goccie si siano formate in un ambicnte in cui, pur essendo il vapor acqueo distribuito inizialmente in modo uniforme, non altrettanto fosse dei nuclei di condensazione, oppure nell'ipotesi che le goccie più grosse derirassero dalla riunione di più goccie minori. Dalla semplice ispezione dei dia-

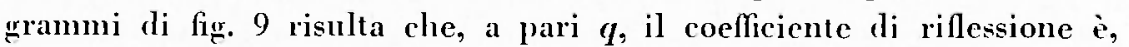
per ogni frequenza, sempre maggiore per quanto minore $\dot{b}$ il raggio delle goccie, salvo per il tratto iniziale allorché $R$ rimane costante e dipendente solo dalla pressione e dalla temperatura dell'ambiente. Ne deriva che hanno effetto predominante nel fissare il coefficiente di riflessione complessivo alle varie frequenze proprio le goccic di raggio minore; cosicché appare presumilile, nelle supposte condizioni, che sulla determinazione del raggio delle goccioline costituenti la nulse non debla influire in maniera perturhatrice la presenza di un piccolo numero di goccie più grrosse.

Diversamente dovelhbe invece concludersi nell'altro caso tipico che le goccie siano distribuite nell'unità di volume del hanco nuvoloso in aliquote di ugual numero $N$ per ogni diverso raggio $a$. In questo caso una valutazione sommaria del coefficiente di riflessione complessivo è immediata per i campi di frequenze sonore in cui valgono 
le relazioni di proporzionalità [15] e [17]: risulta infatti che nella sovrapposizione dei coefficienti di riflessione parzali corrispondenti al. le aliquote di diverso ragrerio prevale il contributo della aliquota di raggio maggiore, che raccoglie d'altra parte in questo caso quasi per intero la massa di acqua liquida contenuta nella nube.

In relazione alle precedenti considerazioni è anche da tencre in delito conto il fatto che le onde sonore della ordinaria gamma udibile da impiegarsi nel presente metodo sono dotate di scarsa direttiviti, e che le formazioni nuvolose si presentano per lo più irregrolari e frastagliate, relativamente diverse dal hanco piano ed indefinito cui il calcolo teorico fa riferimento per le esigenze di una ragionevole semplicità di trattazione. I risultati ottenuti nella pratica applicazione dovranno in grenere essere riferiti non tanto ad un determinato elemento della nube, bensì a tutta la regione, più o meno ampia, investita dall'onda sonora, ed automaticamente ciò porterà a considerare dei valori medi sia per il raggio che per la densità di distribuzione delle groccioline di acqua.

$\mathrm{Ci}$ interessa di vedere anche quali sono i limiti pratici, data la sensilhiliti otteniluile dagli apparecchi, per l'effettivo rilevamento dell'onda riflessa, tenuto conto che, come alhliamo già osservato, il coefficiente di riflessione per attrito è piuttosto piccolo, e dal valore $R_{w^{2}}=6,25.10^{\circ}$ scende sino ai valori $(q / 8)^{-}$. Mostriamo che, usando come strumento di zero all'uscita del ricevitore lo stesso orecchio umano, che è dotato di una elevatissima sensiljilità nella parte centrale dclla gamma di udiloilità, è prevedibile la possibilita di ricevere l'onda riflessa anche con coeflicienti di riflessione assai hassi. Immariniamo dunque che la potenza acustica sia radiata dal trasmettitore, supposto privo di ogni caratteristica direttiva, entro il semispazio delimitato dalla superficie terrestre, cioè entro l'angolo solido 2 r; il riceritore sia invece fornito di un collettore del snono di modeste dimensioni, uno specchio circolare di un metro di raggio, $r=1 \mathrm{~m}$ : il hanco nuroloso riflettente si trovi poi alla quota di $2000 \mathrm{~m}$ e quindi, per incidenza normale, la distanza tra trasmettitore e ricevitore sia $d=4000 \mathrm{~m}$. Lo specchio ricevitore ̀̀ dunque visto dal trasmettitore, riflcsso nella superficie delle nuli, sotto un angrolo solido dato da $\Omega=\pi r^{2} / d^{2}=\pi .10^{-6 / 16}$ : il rapporto tra potenza ricevuta $\Pi_{\mathrm{r}}$ e potenza radiala $W_{t}$ sarà perciò, a parte l'eventuale assorbimento atmosferico, $\Pi_{\mathrm{r}} / \Pi_{\mathrm{t}}=R \Omega / 2 \pi=R \cdot 10^{-4 i} / 32$. Se si ammette una amplificazione in potenza nel ricevitore di $10^{\circ}$ volte, e si parte da una potenza tra- 
smessa di 100 watt, si ha una potenza all'uscita del ricevitore data da $10^{\circ} . R \cdot 10^{-6} . F_{\mathrm{t}}^{*} / 32 \propto 3.10^{* *} R$.

Con un coefficiente di riflessione anche hasso, ad esempio $10^{-10}$, (per $q=10^{-7}$ è $R_{0,1}=6,25.10^{-10}$ ), si ha la potenza utile di $3.10^{-13}$ watt, largamente superiore a quella della soglia di udililita dellorechio umano che si riliene dell'ordine di $10^{-15}$ wall $/ \mathrm{cm}^{-2}$ alla frequenza di circa $2000 \mathrm{p} / \mathrm{s}$. Alle frequenze più basse lale soglia, come ì noto, reresce noterolmente; perì in questo campo, in cui ommi domina il fenomono termodinamico, $\dot{e}$ anche più forte $i l$ coefficiente di riflessione. Invece la potenza riflessa potrebbe essere insufficiente per la rivelacione alle frequenze prossime al linite superiore di udililiti, ove verreble a cadere la frequenza di taylio per attrito per groce relativamente piccole.

Vogliamo da ultimo accennare alle possibiliti che il nostro metodo di sondagrio acustico delle nubi presenta per lo studio della evoluzione nel tempo degli elementi costitutivi di queste, cioe del raggio e del numero delle groce od anche della quantiti di acqua liquida contenutavi; a prescindere naturalmente dalla ovia possibilita di de. dure questa conoscenza da successive determinazioni slatiche in hase ai procedimenti già esaminati.

Per via abbastanza semplice e diretta l'indagine può condursi a partire dalle stesse cisrcostanze da noi precedentemente acquisite: purché si supponga che la disposizione sperimentale sia alta a fornire con continuti on a brevi intervalli di tempo la registrazione della funzione $R(1)$ in un campo di frequenze comprendente la frequenza di taglio per attrito ed almeno una delle due rewioni contigue: appare allora immediato come possa seguirsi levoluzione delle groccioline costituenti lo strato in eui la riflessione ha luogo, considerando la dipendenza della frequenza di tawlio per attrito dal solo raggio delle groce secondo la [10], e la dipendenza del coefficiente comples. sivo di riflessione dalla grandeza q/wa- secondo le [1+], $|16|$, oppure dalla aN/(1) secondo le [15], [17], nei tratli in cui la relativa curva ha, nella rappresentazione logarimica, andamento sensibilnente reltilineo. Cosi ad esempio la circostanza tipica che aumentasse il ragrio $a$, rimanendo costante la fuantita $q$ di acqua liquida contenuta per la semplice riunione di più goceioline, si paleserelbe con una diminuzione della frequenza di taglio per attrito, secondo la $|10|$, e contemporaneamente con una diminuzione del coefficiente di riflessione per 
ciascuna frequenza per cui valgono le $[14],[16 \mid$ ai sensi delle medesime formule. Se invece si avesse ammento del ragrio delle groce per ulteriore condensazione di vapor acqueo sulle medesime, rimanendone costante il numero $N$, si arrebbe ugualmente una diminuzione della frequenza di tagrlio per attrito, ma un aumento del coefficiente di riflessione secondo le formule [15], [17].

Più semplicemente però il rilevamento della evoluzione degrli elementi costituenti le nubi può effettuarsi seguendo, nel diagramma della funzione $R((n)$, la migrazione del punto rappresentativo del coefficiente di riflessione $R\left((1)_{a}\right) \simeq R_{m a} / 2=q^{2} \varepsilon^{2} / 32$ alla pulsazione di taglio per attrito $\omega_{n}=9 v / 2 \varepsilon a^{2}$. Tale punto appare facilmente individuaibile sia perché delimita all'incirca un'estremita della regione di transizione, compresa tra la ()$_{t}$ e la $\left(n_{n}\right.$, sia perche la curva $R(1)$ ) conserva con poche alterazioni, come ripetuamente si disse, la sua forma caratteristica pur spostandosi nel piano rappresentativo al variare dei parametri $a, r, N$.

E ovvio che nel caso in cui il rarrio delle rroccioline aumentasse o diminuisse per riunione o suddivisione delle medesime, rimanendo costante la grandezza q, cioè senza contemporanea evaporazione o condensazione di acqua, il punto $R\left(\left(1_{n}\right)\right.$, (1) si sposterehbe orizzontalmente nel diagramma rappresentativo verso sinistra o rispettivamente verso destra. Nellipotetico caso che si creassero invece nuove roce delle stesse dimensioni delle gia esistenti, per condensazione su nuovi nuclei, oppure che alcune di queste ultime renissero a distruggersi, ciò̀ che arverasse la singolare eventualità della variazione di $q$, e quindi di $N$, rimanendo il raggio a costante, il punto rappresentativo verreblse a spostarsi in direzione verticale verso l'alto o rispettivamente rerso il basso.

Ma il caso più interrsante, perché più vicino alle circostanze comuni. i che si ablia condensazione od evaporazione di acqua sulle gocce esistenti, cioe che si verifichi variazione del raggio $a$, e quindi della quantita $q$, rimanendo costante il numero $X$ delle gocce stesse. Per determinare in tale condizione il loogo del punto rappresentativo $R\left(\left(t_{n}\right)\right.$, (1, basta eliminare a e $q$ fra le due precedenti c la $[13]$; si ettiene

$$
R \omega^{3}=\left(\frac{9 \pi}{4} N\right)^{2} \frac{v^{3}}{\varepsilon},
$$

od anche, prendendo i logaritmi,

$$
\log R+3 \log (t)=\text { cost. }
$$


Nella rappresentazione logaritmica tale luogo è dunque una retta (fig. 10) che passa per il punto inizialmente considerato ed inclinata in modo tale che sia $\operatorname{tg} \beta=3$. Al variare della costante si ha poi na-

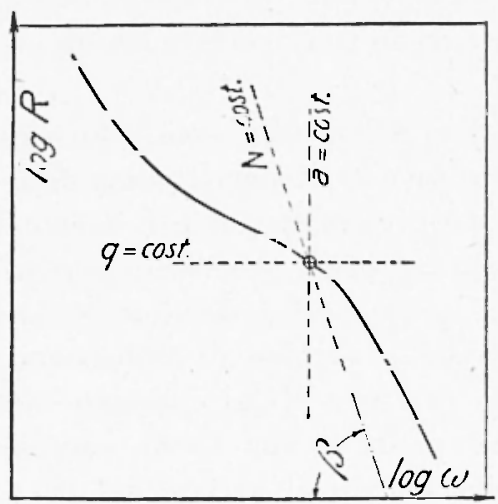

Fig. 10 turalmente una famiglia di rette parallele corrispondenti ai diversi lavori di $N$.

Se quindi dall'esame comparativo delle registrazioni sperimentali in scala logaritmica risultasse con il passare del tempo una traslazione nella direzione ora stabilita del punto $R\left(\omega_{u}\right), \omega_{u}$, e quindi anche di tutta la curva $R((1)$ ), sarebbe logico ritenere che si verificasse nella nube una variazione della quantiti di acqua rimanendo costante il numero delle gocce: precisamente un ingrossamento delle stesse, se la curva $R((0)$ si sposta verso l'alto, e viceversa un impiccolimento se si sposta verso il basso. Se invece dovesse riscontrarsi una traslazione pressoché orizzontale si dovrebbe attrihuirla, come già si disse, alla riunione od alla suddivisione delle gocce, rimanendo all'incirca costante la quantita totale di acqua liquida contenuta nella nube.

Per riassumere i risultati cui ci hanno condotto le considerazioni teoriche esposte nel presente lavoro possiamo conchdere che il metodo di esplorazione acustica delle nubi da noi proposto permette di desumere dal rilevamento del coefficiente di riftessione alle varie frequenze della gamma udibile, la grandezza ed il numero delle goccioline costituenti la nube, ció la quantità di acqua liquida contenutavi nell'unità di volume, nonché le relative eventuali variazioni al trascorrere del tempo. Per quanto solo l'esperienza possa effettivamente accertare i limiti e la potenza del metodo, ci sembra tuttavia lecito prevederne una pratica applicalilità, sopratulto considerando due circostanze caratteristiche: la semplicità dell'interpretazione dei dati sperimentali basata essenzialmente sull'esame della forma e degli spostamenti delle curve del coefficiente di riflessione in funzione della frequenza sonora; la pressoché completa indipendenza dei risultati, legati quasi tutti al fenomeno meccanico dell'attrito opposto al moto delle 
goccioline di acqua dalla viscosità dell'aria, dalle condizioni ambienti di temperatura e di pressione.

$$
\text { Roma - Istituto Nazionale di Geofisica - Settembre } 1951 .
$$

\section{RIASSUNTO}

Si espone e si discute un metodo per l'esplorazione acustica delle nubi, idoneo a determinare il numero delle goccioline di acqua conte. nute per unita di volume ed il loro raggio. Il metodo si basa sulla ri. flessione delle onde sonore della gamma udibile per l'azione simultanea del fenomeno meccanico di trascinamento delle goccioline nello spostamento sonoro, e di quello termodinamico di alterna condensazione ed evaporazione delle medesime per effetto delle oscillazioni di pressione acustica. Nel lavoro si determina il coefficiente di riflessione acustico di un banco di nubi, o si mostra che tale coefficiente dipende in modo caratteristico dalla frequenza sonora, ed ì funzione del raggio o del numero delle goccioline, oltre che della temperatura e della pressione; tuttavia l'influenza di questi ultimi due parametri puo ridursi notevolmente scegliendo opportunamente certi valori delle frequenze sonore d'osservazione, corrispondenti a punti caratteristici in cui muta l'anda. mento del coefficiente di riflessione. Per quanto solo l'esperienza possa accerture i limiti e la potenzialità del metodo proposto, è lecito prevederne una pratica applicazione, specialmente in considerazione del fatto che linterpretazione dei risultati is sostanzialmente fondala sul semplice esame della forma delle curve del coefficiente di riflessione in funzione della frequenza sonora, dalla quale forma può desumersi il raggio delle goccioline che operano la riflessione, e può anche stimarsi il loro numero. Inoltre mediante l'osservazione degli spostamenti che dette curve subiscono al trascorrere del tempo può seguirsi l'evoluzione degli elementi costituenti la nube in conseguenza degli eventi meteorologici.

\section{SLMM.ARY}

A method is described and discussed for the acoustic exploration of clouds, which enables to determine the number of uater droplets which are contained in the volume unit as woll as their radius. This method is based on the reflection of acoustic waves within the audible 
range oucing to the simultaneons action of both the mechanical dragging effect of the water droplets following the acoustic displacement, and the thermodynamic effect of alternative condensation and evaporation of the droplets caused by the escillation of the acoustic pressure. The acoustic reflection coefficient of a cloud bank has been delermined; such a coefficient depends in characteristical manner upon the acoustic frequency, as well as on the radius and number of the droplets, and further on temperature and pressure: the influence of these two last parameters can be however considerabiy reduced by a suitcible choice, for the observation, of some acoustic frequency values which correspond to peculiar points where the behaviour of the reflection coefficient changes.

Thongh limits and possibilities of the proposed method can be ascertained only by experimentation, its practical applications can be reasoncibly foreseen, owing particularly to the fact that the interpretation of the results is substantially based on the mere examination of the form of the reflection coefficient curves against the acoustic frequency, as, from the form of these curves, the radius of the reflection operating droplets can be deduced, and their number can be estimated. Further, through the observation of the above mentioned curves during the elapsing of time, it is possible to follow the changes of the clements which form the clouds related meteorogical events.

\section{BIBLIOGR:AFIA}

(1) Annali di Geofisica 4, 453, 1951.

(2) Phys. ZS. 42, 365, 1941.

(3) Annali di Geofisicai 3, 289, 1950. 